\title{
A Deoxynivalenol-Activated Methionyl-tRNA Synthetase Gene from Wheat Encodes a Nuclear Localized Protein and Protects Plants Against Fusarium Pathogens and Mycotoxins
}

\author{
Dong-Yun Zuo, Shu-yuan Yi, Rong-Jing Liu, Bo Qu, Tao Huang, Wei-Jie He, Cheng Li, He-Ping Li, and Yu-Cai Liao \\ All authors: Molecular Biotechnology Laboratory of Triticeae Crops, first, third, fifth, sixth, and eighth authors: College of Life Science and \\ Technology; second, fourth, seventh, and ninth authors: College of Plant Science and Technology, and ninth author: National Center of Plant \\ Gene Research (Wuhan), Huazhong Agricultural University, Wuhan 430070, People's Republic of China. \\ Accepted for publication 9 February 2016.
}

\begin{abstract}
Zuo, D.-Y., Yi, S.-Y., Liu, R.-J., Qu, B., Huang, T., He, W.-J., Li, C., Li, H.-P., and Liao, Y.-C. 2016. A deoxynivalenol-activated methionyl-tRNA synthetase gene from wheat encodes a nuclear localized protein and protects plants against Fusarium pathogens and mycotoxins. Phytopathology 106:614-623.

Fusarium graminearum is the fungal pathogen that causes globally important diseases of cereals and produces mycotoxins such as deoxynivalenol (DON). Owing to the dearth of available sources of resistance to Fusarium pathogens, characterization of novel genes that confer resistance to mycotoxins and mycotoxin-producing fungi is vitally important for breeding resistant crop varieties. In this study, a wheat methionyl-tRNA synthetase (TaMetRS) gene was identified from suspension cell cultures

treated with DON. It shares conserved aminoacylation catalytic and tRNA anticodon binding domains with human MetRS and with the only previously characterized plant MetRS, suggesting that it functions in aminoacylation in the cytoplasm. However, the TaMetRS comprises a typical nuclear localization signal and cellular localization studies with a TaMetRS::GFP fusion protein showed that TaMetRS is localized in the nucleus. Expression of TaMetRS was activated by DON treatment and by infection with a DONproducing $F$. graminearum strain in wheat spikes. No such activation was observed following infection with a non-DON-producing $F$. graminearum strain. Expression of TaMetRS in Arabidopsis plants conferred significant resistance to DON and $F$. graminearum. These results indicated that this DON-activated TaMetRS gene may encode a novel type of MetRS in plants that has a role in defense and detoxification.
\end{abstract}

Fusarium head blight (FHB) of wheat, barley, and oats, and Gibberella ear rot (GER) of maize are globally important crop diseases caused by the fungal pathogen Fusarium graminearum (anamorph: Gibberellazeae (Schwein.) Petch). FHB and GER epidemics not only reduce yields, but also dramatically affect crop quality due to the presence of mycotoxins from the pathogen in the harvested grains (Bai and Shaner 2004; Kazan et al. 2012; Li et al. 2010a; Xu and Nicholson 2009). Natural plant resistance to $F$. graminearum is currently inadequate, and most wheat, barley, and maize cultivars currently grown globally are susceptible to this fungal pathogen. FHB epidemics in wheat and barley occur frequently in many regions in China (Qu et al. 2008; Yang et al. 2008) and this disease has re-emerged as a serious threat to agriculture in Europe and North America since the 1990s, resulting in massive economic impacts (Bai and Shaner 2004; Kazan et al. 2012; McMullen et al. 1997; Scherm et al. 2013). Between 1991 and 1997, losses caused by FHB in wheat and barley in the United States alone were estimated to be about $\$ 4.8$ billion (Johnson et al. 2003).

The predominant mycotoxins present in cereal grains infected by $F$. graminearum are trichothecenes deoxynivalenol (DON), 3-acetyldeoxynivalenol (3-ADON), and 15-acetyldeoxynivalenol (15-ADON) (Placinta et al. 1999; Zhang et al. 2013). DON is a potent inhibitor of protein translation that targets the 60S ribosomal protein L3 at the peptidyltransferase center and induces apoptosis in

Corresponding authors: H.-P. Li; E-mail address: hepingli@mail.hzau.edu.cn, Y.-C. Liao; E-mail address: yucailiao@mail.hzau.edu.cn

*The $\boldsymbol{e}$-Xtra logo stands for "electronic extra" and indicates that two supplementary figures are published online.

http://dx.doi.org/10.1094/PHYTO-12-15-0327-R

(C) 2016 The American Phytopathological Society eukaryotes (Fried and Warner 1981; Rocha et al. 2005). DON also acts as a virulence factor in plants, promoting the spread of fungi in plant tissues (Bai et al. 2002; Desjardins et al. 1996). DON is highly toxic to humans and domestic animals; consumption of DONcontaminated cereals by domestic animals or humans can cause several chronic effects such as immune dysregulation, anorexia, and growth retardation, as well as acute poisoning leading to emesis and diarrhea (Goswami and Kistler 2004; Pestka 2010; Rocha et al. 2005). The regulatory limit of DON set by the WHO/FAO Expert Committee on Food Additives in 2001 for original grain or wheat flour ranges from 0.70 to $1.2 \mathrm{mg} \mathrm{kg}^{-1}$ (Food and Agriculture Organization 2004). A recent study reported that only 2 of 14 wheat grain samples harvested from commercial wheat fields in FHB epidemic regions in China had DON levels lower than the regulatory limit for consumption (Zhang et al. 2013).

Several genes coding enzymes that modify or detoxify DON have been identified, and some of these have been used to improve tolerance to DON in plants (Ito et al. 2013; Li et al. 2010b; Poppenberger et al. 2003). For instance, one UDP-glycosyltransferase encoded by the DOGT1 gene from Arabidopsis thaliana was shown to detoxify DON by catalyzing the transfer of a glucose moiety from UDP-glucose to the C3 hydroxyl group of DON; constitutive expression of this UDPglycosyltransferase in Arabidopsis thaliana led to enhanced tolerance against DON (Poppenberger et al. 2003). Heterologous expression of a barley UDP-glucosyltransferases (HvUGT13248) in yeast protected the yeast cells against DON (Schweiger et al. 2010); this barley gene and a homolog from wheat were both shown to increase tolerance to DON in Arabidopsis thaliana (Shin et al. 2012). Wheat plants expressing an F. sporotrichioides-derived gene (FsTRI101) encoding an enzyme that transfers an acetyl moiety to the $\mathrm{C} 3$ hydroxyl group of DON showed both a reduction in DON accumulation and increased resistance against F. graminearum (Okubara et al. 2002). 
Methionyl-tRNA synthetase (MetRS) is conventionally considered to catalyze the aminoacylation of a specific amino acid, methionine, with cognate tRNA to form Met-tRNA ${ }_{i}{ }^{\text {Met }}$ during protein translation; Met-tRNA ${ }_{i}$ Met is a key factor in translation initiation (Havrylenko et al. 2010). Interestingly, various studies have shown that MetRS in eukaryotes has other important functions. MetRS may play a role in the biogenesis of rRNA (Ko et al. 2000). Under conditions of oxidative stress, MetRS has been shown to charge Met residues onto noncognate tRNAs at a high frequency (Netzer et al. 2009). Mammalian MetRS displays tumor suppressor activity (Kwon et al. 2011). Furthermore, MetRS has been identified as an important target for the development of antiparasitic drugs active against human African trypanosomiasis caused by the protozoan Trypanosoma brucei. Expression of the TbMetRS gene in T. bruce i is activated by the MetRSspecific inhibitors, and the activated expression of the TbMetRS in turn confers resistance to the inhibitors (Ranade et al. 2013).

To date, surprisingly, only one MetRS from plants has been characterized in detail. This lack of attention probably owes to the fact that both the canonical function in protein translation and the attendant fundamental importance of MetRS proteins are already widely understood and appreciated in biology. The detailed plant MetRS study investigated a rice MetRS that has a canonical function acting as cis-acting factor for aminoacylation. Interestingly the C-terminal region of this rice MetRS protein has an endothelial monocyte activating polypeptide II-like domain that increases the catalytic efficiency of the aminoacylation reaction (Kaminska et al. 2000).

In this study, we used suppression subtractive hybridization (SSH) to identify a MetRS gene from DON-treated wheat suspension cell cultures. The expression patterns of the MetRS gene were evaluated in the spikes of two different wheat varieties following treatment with DON and following inoculation with either a DONproducing $F$. graminearum strain or a mutant $F$. graminearum stain that is not able to produce DON (a strain carrying a disrupted Tri5 gene, Tri5- strain). Our objectives were to clone and characterize this wheat MetRS gene, and to understand whether or not it confers tolerance to DON and/or resistance to $F$. graminearum in plants. Our results include the novel finding that this wheat MetRS gene codes for a nuclear localized protein that can protect transgenic Arabidopsis plants against FHB pathogens and the associated mycotoxin DON. Therefore, this wheat MetRS gene represents an exciting new potential source of genetic resistance to FHB and GER. Further, this gene can be used in subsequent basic investigations seeking to characterize potentially novel plant defense and detoxification mechanisms.

\section{MATERIALS AND METHODS}

Biological materials. Arabidopsis thaliana 'Columbia' and two wheat cultivars were used in this study; the Sumai3 (S3) wheat cultivar has resistance to F. graminearum, and the Zheng9023 (Z9) cultivar is susceptible to this fungal pathogen (Cheng et al. 2015). Wheat plants were grown in pots $(12 \times 14 \mathrm{~cm})$ that were kept in a growth chamber under conditions of $16 / 8 \mathrm{~h}$ day/night at 18 to $20^{\circ} \mathrm{C}$. Arabidopsis plants were grown according to standard laboratory practices (Weigel and Glazebrook 2002). F. graminearum strain 5035, which was isolated from a scabby wheat spike in Wuhan, China (Qu et al. 2008), and a mutant strain Tri5- carrying a Tri5deletion in the background of $F$. graminearum 5035 (Li et al. 2010b) were cultured on potato dextrose agar (PDA). Suspension cell cultures were generated from seedlings of wheat $\mathrm{Z} 9$ and cultured at $22^{\circ} \mathrm{C}$ with shaking at $150 \mathrm{rpm}$ as described previously (Fellers et al. 1995). Agrobacterium tumefaciens strain GV3101, the pTRA vector, and the $G F P$ (green fluorescence protein) gene were handled as described previously (Peschen et al. 2004). A biolistic gun (PDS$1000 \mathrm{He}$, Bio-Rad) was used for bombardment (Li et al. 2008).

Mycotoxin treatment and fungal inoculation of wheat spikes. Both the inoculation experiments and the DON treatment of wheat spikes were carried out as previously described (Li et al. 2010b).
Briefly, a macroconidial suspension of $F$. graminearum strain 5035 and the mutant strain Tri5- was adjusted to a concentration of $5 \times$ $10^{5}$ spores $\mathrm{ml}^{-1}$. At the midflowering point, 20 spikes of each cultivar, with a single spike per plant, were inoculated with either a $10-\mu \mathrm{l}$ droplet of the macroconidia or $15 \mu \mathrm{l}$ of DON $\left(1.5 \mathrm{mg} \mathrm{ml}^{-1}\right)$ in $0.2 \%$ Tween 20 that were prepared as previously described (Li et al. 2010b). A $0.2 \%$ Tween 20 solution (in water) was used as the mock inoculation treatment. Spikes were injected with a pipette tip to the central floret of one middle spikelet (Li et al. 2008). After inoculation, the plants were kept in conditions of high humidity for 3 days, and then grown in a green house. The fungal inoculated spikes were collected at $24,48,72$, and $96 \mathrm{~h}$ postinoculation (hpi), while the DON-treated spikes were harvested at 4, 12, 24 and $48 \mathrm{~h}$ posttreatment (hpt). TaMetRS gene expression in these samples was then analyzed with quantitative real-time polymerase chain reaction (qRT-PCR).

RNA extraction and suppression subtractive hybridization. Immediately following transfer to fresh media, 7-day-old cell cultures were treated with DON at $50 \mathrm{mg} \mathrm{liter-1}^{-1}$ (Sigma-Aldrich) or water (control) (Li et al. 2010b), and the treated cells were harvested at 4, 12, and 48 hpt. Total RNA samples isolated from the DONtreated suspension cell cultures of wheat Z9 collected at different time intervals were pooled equally and used as the tester. Similarly, total RNA samples isolated from water-treated control cultures were used as the driver. mRNA was isolated from the tester and driver RNA with a PolyA mRNA isolation kit (Promega, Madison, $\mathrm{MI}$ ), and $2-\mu \mathrm{g}$ aliquots of each mRNA pool were used as the template for the synthesis of double-stranded cDNA performed with a PCR-Select cDNA subtraction kit (Clontech, Mountain View, CA), as previously described (Abebe et al. 2004). The final PCR products from the SSH process were cloned into pGEM-T Easy vectors (Promega).

Cloning and sequence analysis of the TaMetRS gene. A 390-bp cDNA fragment was identified from the SSH (Supplementary Fig. S1) cDNA library and analyzed by BLAST homology searching in the NCBI database (http://blast.ncbi.nlm.nih.gov/ Blast.cgi). This 390-bp sequence was used to design primers to clone the wheat MetRS gene by PCR using a cDNA template from spikes of wheat Z9 sampled at 1 day postinoculation with DON. The missing $5^{\prime}$ and $3^{\prime}$ sequences of the MetRS gene were obtained by rapid amplification of cDNA ends (RACE) using the primers listed in Table 1 and a SMART RACE cDNA Amplification Kit (Takara, Dalian, China). The full-length cDNAs of the TaMetRS genes from wheat Z9 and S3 were obtained by PCR using the TaMetRS-F1 and TaMetRS-R1 primers (Table 1). The PCR products were purified with a nucleic acid purification kit (Axygen, Tewksbury, MA) and cloned into the pGEM-T Easy vector; the nucleotide sequences of the cloned PCR products were then sequenced (Sangon, China). The amino acid sequence deduced from the TaMetRS gene from Z9 (accession number KP713774) was aligned with the MetRS amino acid sequences from additional plant species using the MegAlign program of Bioedit (http://www.softpedia.com/get/Science-CAD/ BioEdit.shtml). A phylogenetic neighbor-joining tree was generated with MEGA software with the neighbor-joining method (Tamura et al. 2011).

Transient expression of the TaMetRS gene. The pTRAGFP vector containing a green fluorescent protein (GFP) and a CaMV35S promoter-nos terminator (Peschen et al. 2004) was used for the transient expression assays in onion epidermal cells, in an effort to determine the subcellular localization of TaMetRS. The open reading frame (ORF) of TaMetRS was amplified using the gene-specific TaMetRS-F2 and TaMetRS-R2 primers (Table 1) that contained a flanking EcoRI site at the 5' end and a BamHI restriction site at the $3^{\prime}$ end. Both the PCR products and the vector were digested with EcoRI and BamHI (Takara) and ligated to generate the fusion construct TaMetRS::GFP. TaMetRS::GFP was then transformed into onion epidermis cells via biolistic gun bombardment; the pTRA-GFP vector was used as a control. Onion epidermis cells were 
placed in the dark for 16 to $48 \mathrm{~h}$ before being treated with a $2 \mathrm{M}$ sucrose solution. Optical and epifluorescence microscopic analysis of the cells was performed with a Nikon eclipse 90i microscope (Nikon, Tokyo, Japan).

Plant transformation. The pTRA-MetRS construct was introduced into Agrobacterium tumefaciens strain GV3101. Arabidopsis thaliana 'Columbia' was transformed via the floral dip method (Zhang et al. 2006). Transgenic plants were selected on half-strength Murashige and Skoog (MS) medium containing kanamycin at $50 \mathrm{mg}$ liter $^{-1}$ and further identified by PCR and RT-PCR with primers in Table 1.

qRT-PCR. Wheat spikes and seedlings inoculated with F. graminearum strain 5035 or the mutant Tri5- strain were harvested at $24,48,72$, and $96 \mathrm{hpi}$, while wheat spikes treated with DON or water were harvested at 4, 12, 24, and 48 hpi. Five spikes for each treatment were harvested and used for RNA isolation. Total plant RNA was isolated using Trizol reagent (Invitrogen, Carlsbad, CA) as previously described ( $\mathrm{Li}$ et al. 2010b). Three replicates were performed. PCR amplification was performed with an iQ5 Cycler (Bio-Rad) with the following thermal cycling program: $95^{\circ} \mathrm{C}$ for $4 \mathrm{~min}$, followed by 40 cycles of $94^{\circ} \mathrm{C}$ for $15 \mathrm{~s}, 62^{\circ} \mathrm{C}$ for $20 \mathrm{~s}$, and $72^{\circ} \mathrm{C}$ for $20 \mathrm{~s}$. The plate read was at $72^{\circ} \mathrm{C}$ for $30 \mathrm{~s}$. A melting curve analysis was performed to evaluate the specificity of each PCR primer pair by incubating the reaction at $95^{\circ} \mathrm{C}$ for $20 \mathrm{~s}$, cooling to $55^{\circ} \mathrm{C}$ for $10 \mathrm{~s}$, and increasing to $95^{\circ} \mathrm{C}$ at a rate of $0.5^{\circ} \mathrm{C}$ per $10 \mathrm{~s}$. Each biological sample was tested in triplicate, and data were normalized to the plant $\beta$-actin gene ( $\mathrm{Li}$ et al. 2010b). Sequence-specific primers TaMetRS-F4/TaMetRS-R4 and Actin-F/Actin-R (Table 1) were designed using the Primer 5 program (Premier Biosoft International, Palo Alto, CA).

Southern blotting. Genomic DNA from leaves of wheat Z9 or Arabidopsis thaliana plants was isolated with the CTAB method, as described previously ( $\mathrm{Li}$ et al. 2008). A total of $15 \mu \mathrm{g}$ of DNA from each wheat sample was digested with 60 units of EcoRI, HindIII, or SacI. Five micrograms of DNA from each Arabidopsis sample was digested with 20 units of SacI. All digestions were performed overnight at $37^{\circ} \mathrm{C}$. The digested DNA was electrophoresed on $0.8 \%$ agarose gels. DNA fragments were transferred onto a Hybond-N+ nylon membrane (Amersham, UK) and then hybridized with $\alpha-\left[{ }^{32} \mathrm{P}\right]-\mathrm{dCTP}$-labeled DNA fragment of the TaMetRS gene sequence (877 bp of genomic sequence, from nucleotide 1 to 877 ) amplified with the TaMetRS-F3 and TaMetRS-R3 primers (Table 1) (Li et al. 2008). Autoradiographs were recorded and analyzed with a BAS1800-II imaging analysis system (Fuji Film, Tokyo, Japan).

DON tolerance assays. Seeds of T2 transgenic plants and nontransgenic control were surface-sterilized and placed on halfstrength MS solid medium supplemented with kanamycin at

TABLE 1. Names and sequences of primers used in this study

\begin{tabular}{|c|c|}
\hline Primer & Sequence $\left(5^{\prime}-3^{\prime}\right)$ \\
\hline 5'RACE-F1 & $\begin{array}{l}\text { CTAATACGACTCACTATAGGGCAAGCAGTG } \\
\text { GTATCAACGCAGAGT }\end{array}$ \\
\hline 5'RACE-R1 & GCCCTTGCGTTTCTTTCGGCCTGACTG \\
\hline 5'RACE-F2 & AAGCAGTGGTATCAACGCAGAGT \\
\hline 5'RACE-R2 & GCCTGCAAATGTTTCCCTGAAGCTATCC \\
\hline 3'RACE-F1 & GCCGATGAAAAGGGGGAGAGTGACAAGG \\
\hline 3'RACE-R1 & GCTGTCAACGATACGCTACGTAACG \\
\hline 3'RACE-F2 & GGCGAAAAGGCCTTGGGATCTTATACC \\
\hline 3'RACE-R2 & CGCTACGTAACGGCATGACAGTG \\
\hline TaMetRS-F1 & AAACGCTGAAAGGAAGAAATACACG \\
\hline TaMetRS-R1 & GGCGGAGAGATAGAAAAGTAAGAGT \\
\hline TaMetRS-F2 & GATGAATTCATGTCGTCGGGGAAGGGGGA \\
\hline TaMetRS-R2 & AGCGGATCCGTTAGCCCTTGCGTTT \\
\hline TaMetRS-F3 & AAACGCTGAAAGGAAGAAATACACG \\
\hline TaMetRS-R3 & CTTTCAAACTTGATAGTGCAGC \\
\hline TaMetRS-F4 & GTGCCTTTGTTCACAGAGTT \\
\hline TaMetRS-R4 & GTTTTGCAGCTTCCAGTTAG \\
\hline Actin-F & CAGCAATGTATGTCGCAATC \\
\hline Actin- $\mathrm{R}$ & TAGCATGAGGAAGCGTGTAT \\
\hline
\end{tabular}

$50 \mathrm{mg}$ liter-1. The seeds were kept at $4^{\circ} \mathrm{C}$ in the dark for 2 days; the seedlings were then grown for 2 weeks with a $16 \mathrm{~h} / 8 \mathrm{~h}$ light-dark period at 22 to $24^{\circ} \mathrm{C}$. T2 transgenic and nontransgenic control seedlings with the same (indistinguishable between the genotypes) morphology and growth vigor were transferred to half MS media supplemented with different DON concentrations $(0,5,10$, and $15 \mathrm{ppm}$ ) (Ma et al. 2010). Seedlings of 20 plants for each genotype were grown for 2 weeks, and their root lengths and total biomasses (fresh weight) were measured. These experiments were performed in triplicate.

Fungal resistance assays. After germination and growth for 2 weeks on MS medium in Petri dishes, as described above, T2 transgenic Arabidopsis thaliana plants and nontransgenic control plants were transplanted to soil and grown for 5 weeks in a growth chamber. The plants were then sprayed once with an $F$. graminearum strain 5035 conidiospore suspension $\left(1 \times 10^{5}\right.$ spore $\left.\mathrm{ml}^{-1}\right)$ (Urban et al. 2002). The inoculated plants were kept in growth chambers with high relative humidity. Twenty plants for each genotype were used for each inoculation, and the experiment was performed in triplicate. Disease severity, expressed as a Fusarium-Arabidopsis disease value (FAD value), was scored at both 7 and 10 days after inoculation, as described previously (Urban et al. 2002).

Statistical analysis. All data were analyzed using SAS 6.12 software (SAS Institute, Cary, NC). Inferential statistical tests used significance levels of 0.05 or 0.01 , as indicated in the figures and text.

\section{RESULTS}

Cloning of TaMetRS and phylogenetic analysis. SSH was used to produce 100- to 500-bp gene fragments corresponding to mRNAs found only in DON-treated cell cultures derived from wheat Z9 (i.e., not present in control samples). Dot blot analyses of 960 clones induced by DON identified 118 gene fragments that were profoundly up-regulated; one of them, designated as TaMetRS, was selected for further analysis owing to its strongly induced expression following DON treatment. This gene fragment was $390 \mathrm{bp}$ in length, and a BLAST (Altschul et al. 1997) analysis indicated that this fragment shared homology with methionyl-tRNA synthetase (MetRS) genes in the NCBI database. 3'-RACE and 5'-RACE of Z9 were used to generate 501- and 1,858-bp fragments, respectively. These two fragments were used to assemble a sequence of $2,186 \mathrm{bp}$ in total length, which contained an ORF encoding 596 amino acids. The genomic sequence of the TaMetRS gene is 3,905 bp in length and has seven introns. Wheat Z9 and S3 contain identical sequences of the amino acids and genomic sequence of the TaMetRS gene.

The molecular weight of the polypeptide deduced from the TaMetRS sequence was estimated to be $67.07 \mathrm{kDa}$, and the $\mathrm{pI}$ value was predicted to be 5.78. As expected, TaMetRS shares higher sequence similarity with its homologs from rice $(76 \%$; Kaminska et al. 2000) and human (53\%; Kwon et al. 2011) than with homologs from other organisms such as Saccharomyces cerevisiae (49\%), Escherichia coli (27\%), and Trypanosoma brucei (24\%). Motif sequence searching and multiple alignments indicated that TaMetRS contains typical domains that have been functionally characterized in other MetRS proteins (Fig. 1). TaMetRS has an N-terminal and a central catalytic core domain that contains a class I HIGH motif and a KMSKS motif. The functionality of the catalytic core domain is based on a Rossman fold, and is responsible for the ATP-dependent formation of enzyme bound aminoacyl-adenylate. The HIGH and KMSKS motifs are highly conserved structures in MetRS proteins and are involved in ATP binding (Koh et al. 2012). A tRNA anticodon-binding domain is located in the $\mathrm{C}$-terminal region adjacent to the catalytic core domain and the former recognizes and specifically binds to the tRNA anticodon. In addition, a nuclear export signal (NES) and a nuclear localization signal (NLS) are present in the TaMetRS sequence (Fig. 1). Thus, the TaMetRS sequence possesses the characteristic domains required for catalyzing the transfer of methionine to the $3^{\prime}$ end of its tRNA. 
Phylogenetic analyses of TaMetRS together with 28 other MetRSs from 10 plant species including six dicotyledons (Arabidopsis thaliana, Populus euphratica, Glycine max, Solanum lycopersicum, Solanum tuberosum, and Nicotiana tomentosiformis) and four monocotyledons (Oryza sativa, Sorghum bicolor, Zea mays, and Brachypodium distachyon), five metazoan (Homo sapiens, Bos taurus, Pan troglodytes, Xenopus laevis, and Pararge aegeria), five fungi (Saccharomyces cerevisiae, Aspergillus fumigatus, Fomitiporia mediterranea, Rhizoctonia solani, and Cladophialophora psammophila), four bacteria (Rhodopirellula europaea, Legionella pneumophila, Salmonella enterica, and Escherichia coli), and four protists (Nannochloropsis gaditana, Trypanosoma brucei, Hammondia hammondi, and Toxoplasma gondii) revealed clearly different main evolutionarily clusters, each of which was comprised of further subclusters (Fig. 2). The wheat MetRS that we identified with the SSH strategy and subsequently cloned, TaMetRS, was separated from the remaining plant MetRSs, suggesting the potential for the presence of unique features in this protein.
Southern blotting was performed with Z9 genomic DNA that was digested with the restriction enzymes EcoRI, HindIII, or SacI and hybridized with a probe containing a HindIII site. The number of fragments detected by Southern hybridization varied between four and eight, suggesting the presence of a low copy number of this gene in the wheat genome.

Expression pattern of the TaMetRS gene in response to DON. To reveal whether TaMetRS gene is activated in planta in response to DON, spikes of wheat Z9 and S3 were treated with DON. RNA was isolated from these samples and analyzed with qRT-PCR. The qRT-PCR results revealed a quick activation of TaMetRS gene expression in wheat spikes following DON treatment. The expression of TaMetRS in Z9 and S3 was increased by up to 43and 22-fold, respectively, at $4 \mathrm{hpt}$. Marked induction was observed in both wheat cultivars at $12 \mathrm{hpt}$; induction peaks in Z9 and S3 up to 700and 600-fold were observed at $24 \mathrm{hpt}$. Later, by the $48 \mathrm{hpt}$ time point, TaMetRS expression had declined to levels lower than those observed at the $12 \mathrm{hpt}$ time point (Fig. 3A).

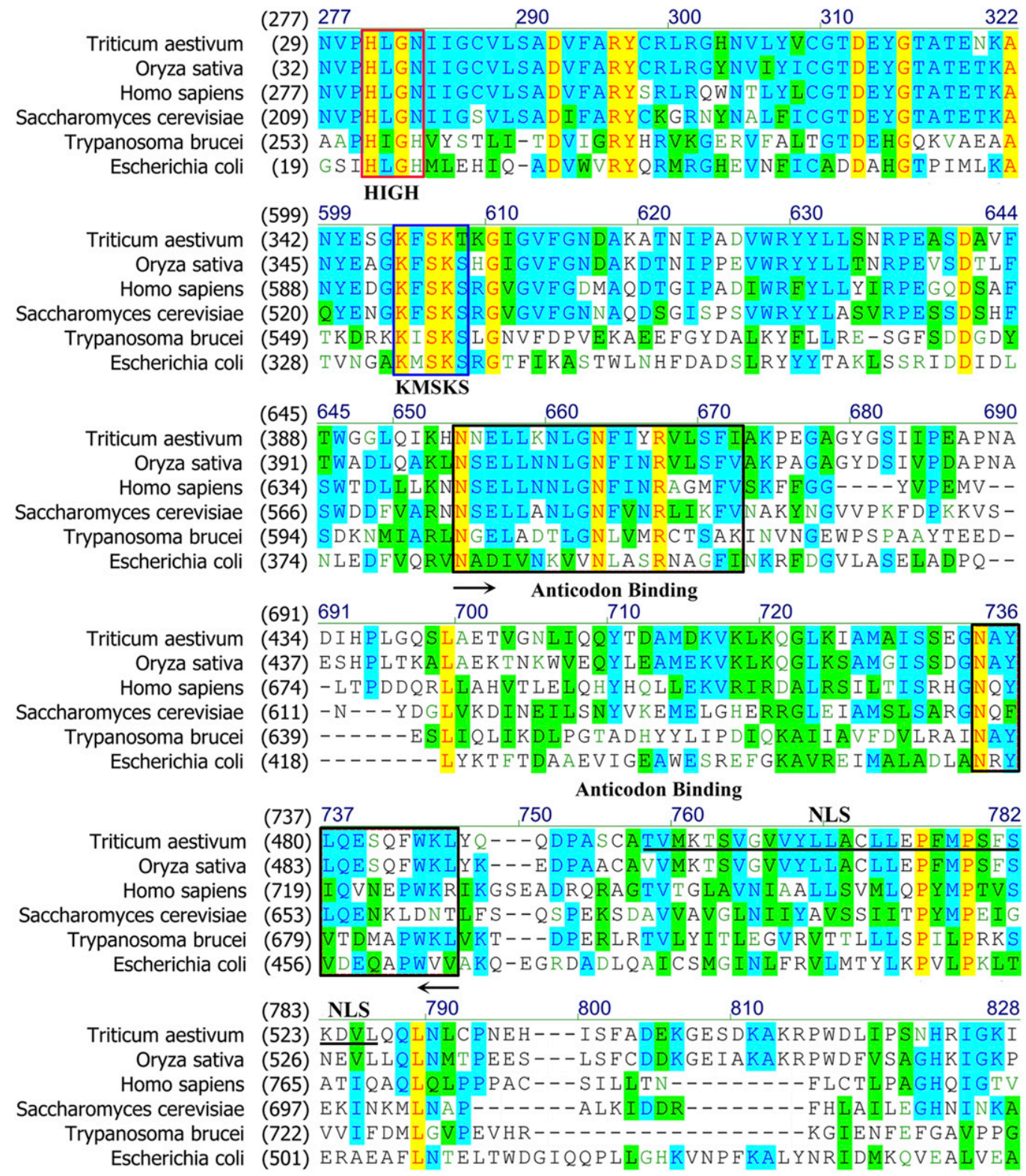

Fig. 1. Multiple sequence alignment of TaMetRS with its homologs from species including Oryza sativa, Homo sapiens, Saccharomyces cerevisiae, Trypanosoma brucei, and Escherichia coli. The conserved domains that are involved in catalytic activity are labeled and marked with red (HIGH) and blue (KMSKS) boxes; the conserved regions for ATP binding and anticodon-binding are indicated by arrows and marked with black boxes; the conserved sequence for nuclear localization signal (NLS) is underlined. 
To characterize the TaMetRS expression patterns in wheat spikes in response to DON produced by $F$. graminearum, Z9 and S3 wheat spikes were inoculated by single floret injection with conidial spores of either the DON-producing $F$. graminearum strain 5035 or the non-DON-producing strain Tri5- (Chen et al. 2011). qRT-PCR expression analysis was performed for inoculated spikes samples collected at 24, 48, 72, and 96 hpi. As is shown in Figure 3B, infection by both Fusarium strains slightly induced the expression of the TaMetRS gene at 24 hpi. Importantly, from 48 to 96 hpi, a significantly different induction pattern was observed in the plants

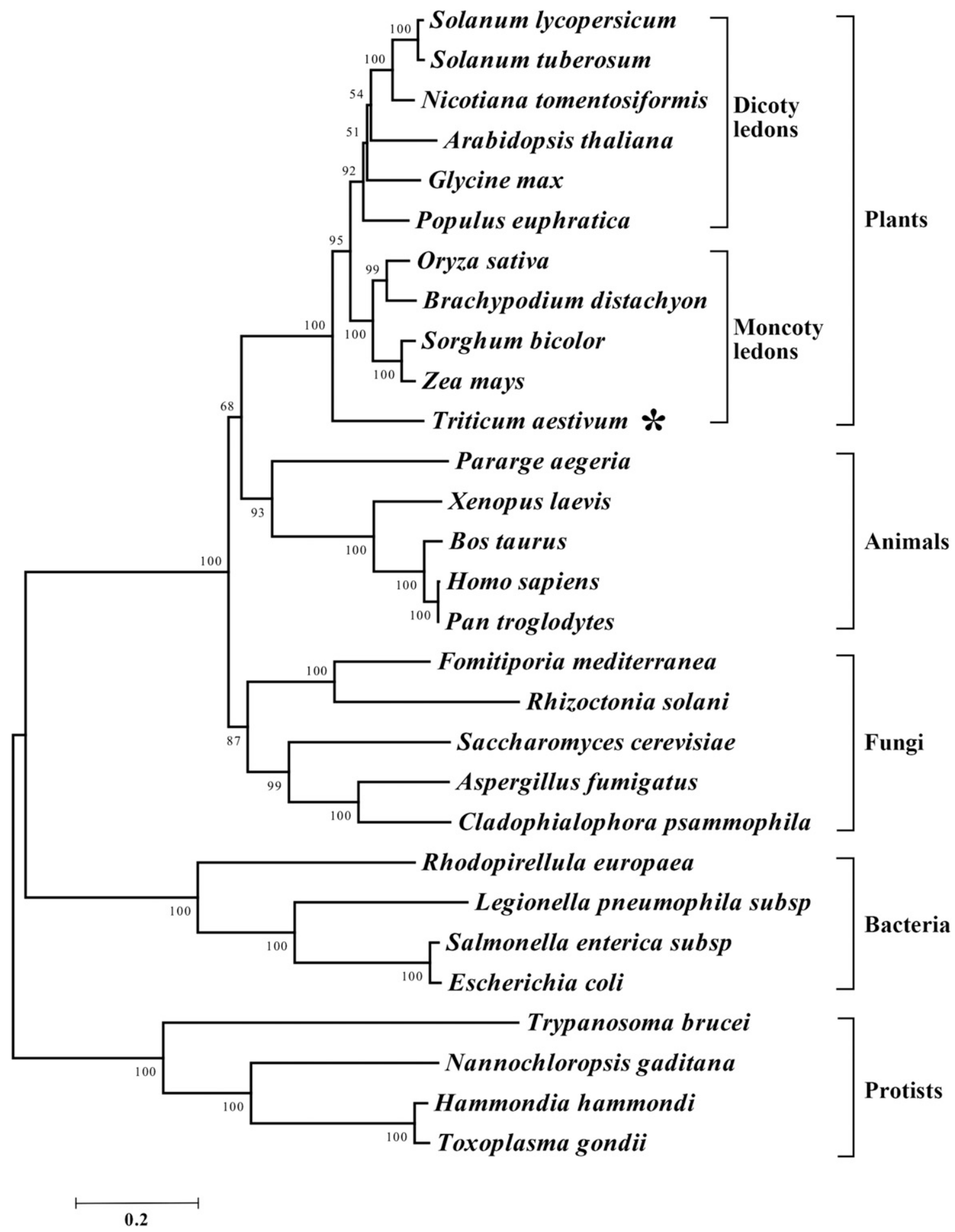

Fig. 2. Phylogenetic tree generated from the deduced methionyl-tRNA synthetase (MetRS) amino acid sequences from Triticum aestivum and 10 other plant species including six dicotyledons: Arabidopsis thaliana (NP_193114), Populus euphratica (XP_011045621), Glycine max (XP_006592017), Solanum lycopersicum (XP_004242831), Solanum tuberosum (XP_006361630), Nicotiana tomentosiformis (XP_009619836) and four monocotyledons: Oryza sativa (AAC99620.1), Sorghum bicolor (EES05588.1), Zea mays (XP_008679089.1), and Brachypodium distachyon (XP_003558495.1); five metazoan species including Homo sapiens (BAA95668.1), Bos taurus (AAI11363.1), Pan troglodytes (JAA37719.1), Xenopus laevis (NP_001086723.1), and Pararge aegeria (JAA87776.1); five fungal species including Saccharomyces cerevisiae (EDN61849.1), Aspergillus fumigatus (KEY81802.1), Fomitiporia mediterranea (XP_007263379.1), Rhizoctonia solani (ELU44219.1), Cladophialophora psammophila (XP_007751852.1); four protist species including Nannochloropsis gaditana (EWM29380.1), Trypanosoma brucei (XP_822407.1), Hammondia hammondi (XP_008889109.1), Toxoplasma gondii (KFH08917.1), and four species of bacterium including Salmonella enterica (YP_005243109.1), Legionella pneumophila (YP_005187204.1), Escherichia coli (EDZ88679.1), Rhodopirellula europaea (WP_008661798.1). Full-length MetRS amino acid sequences from various species were used to construct the phylogenetic tree with the neighbor-joining method. 
inoculated with strain 5035 versus those inoculated with Tri5-. Inoculation of spikelets with the DON-producing strain clearly induced stronger TaMetRS gene expression than did inoculation with Tri5-. This stronger induction of TaMetRS gene expression by the DON-producing fungal strain was observed with both wheat varieties at each of the three time points assayed. Moreover, as infection time increased, there was a steady increase in the strength of the induction (fold increase) of transcripts in spikes inoculated with the $F$. graminearum 5035 strain. Specifically, inoculation with F. graminearum 5035 induced 4.4- to 4.5 -fold increase at $48 \mathrm{hpi}$, 6.8- to 6.9-fold at $72 \mathrm{hpi}$, and 7.6- to 8.7-fold at $96 \mathrm{hpi}$. In stark contrast, the TaMetRS expression levels in wheat spikes inoculated with the Tri5- strain remained consistent at all time points; at no point was there greater than twofold induction of TaMetRS expression relative to the expression of the control $\beta$-actin gene. These results indicated that the DON present in plant tissue produced directly by colonizing Fusarium pathogens induced TaMetRS gene expression at similar magnitudes in different wheat genetic backgrounds, and the strength of the induction increased gradually as increasing duration of infection.
Subcellular localization of TaMetRS. To assess the subcellular localization of TaMetRS in plant cells, a TaMetRS::GFP fusion construct was transformed into onion epidermal cells. As is shown in Figures 4A to $\mathrm{C}$, the green fluorescent signal of the GFP control was present throughout the onion epidermal cells, including in the nucleus, the plasma membrane, and the cytoplasm. The fluorescent signal of the TaMetRS::GFP fusion was clearly observed exclusively in the nucleus (Figs. 4D to F). These results indicate that TaMetRS is a nucleus-localized protein.

DON tolerance in transgenic Arabidopsis expressing TaMetRS. To ascertain whether or not the TaMetRS gene from wheat could confer tolerance to DON in a different plant species, two independent transgenic Arabidopsis thaliana lines expressing the TaMetRS gene, MetRS-1 and MetRS-2, were selected from 15 transgenic lines and their T2 generations were assayed for DON tolerance. The transgenic Arabidopsis plants were identified by PCR and RT-PCR, and the integration of the transgene was verified by Southern blotting (Fig. 5). In the DON tolerance assay of the Arabidopsis plants, a pilot experiment with three different concentrations of DON $(5,10$, and $15 \mathrm{ppm})$ was performed to comparatively assess the
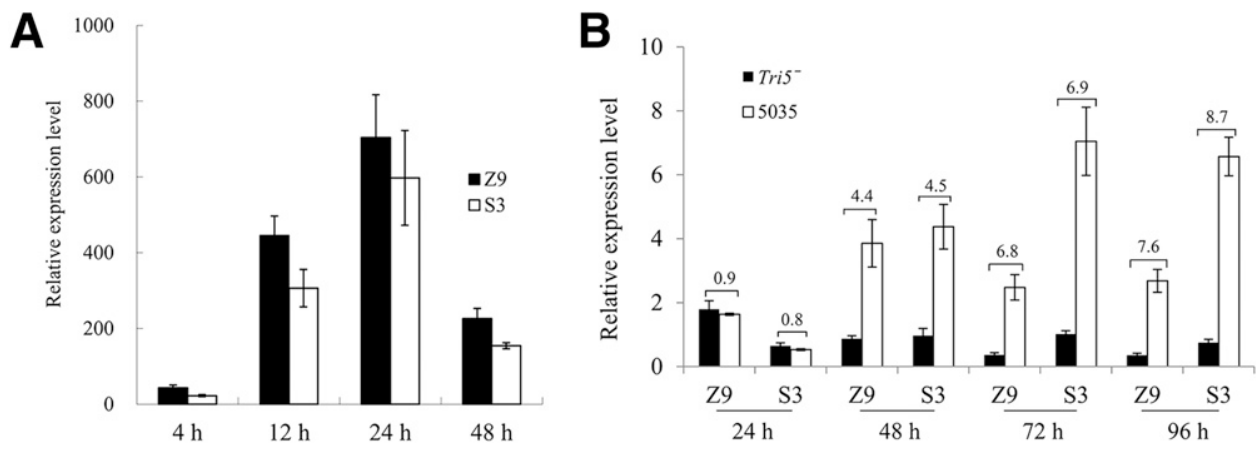

Fig. 3. Expression analyses of the TaMetRS gene in wheat spikes by quantitative real-time polymerase chain reaction. A, RNA extracted from spikes of wheat Zheng9023 (Z9) and Sumai3 (S3) at 4, 12, 24, and $48 \mathrm{~h}$ posttreatment with deoxynivalenol $\left(1.5 \mathrm{mg} \mathrm{ml}^{-1}\right)$. B, RNA extracted from spikes of the two wheat cultivars at 24, 48, 72, and $96 \mathrm{~h}$ postinoculation with Fusarium graminearum 5035 (5035) or the Tri5- mutant strain (Tri5-). Relative gene expression levels were calculated relative to the expression level of the $\beta$-actin gene.
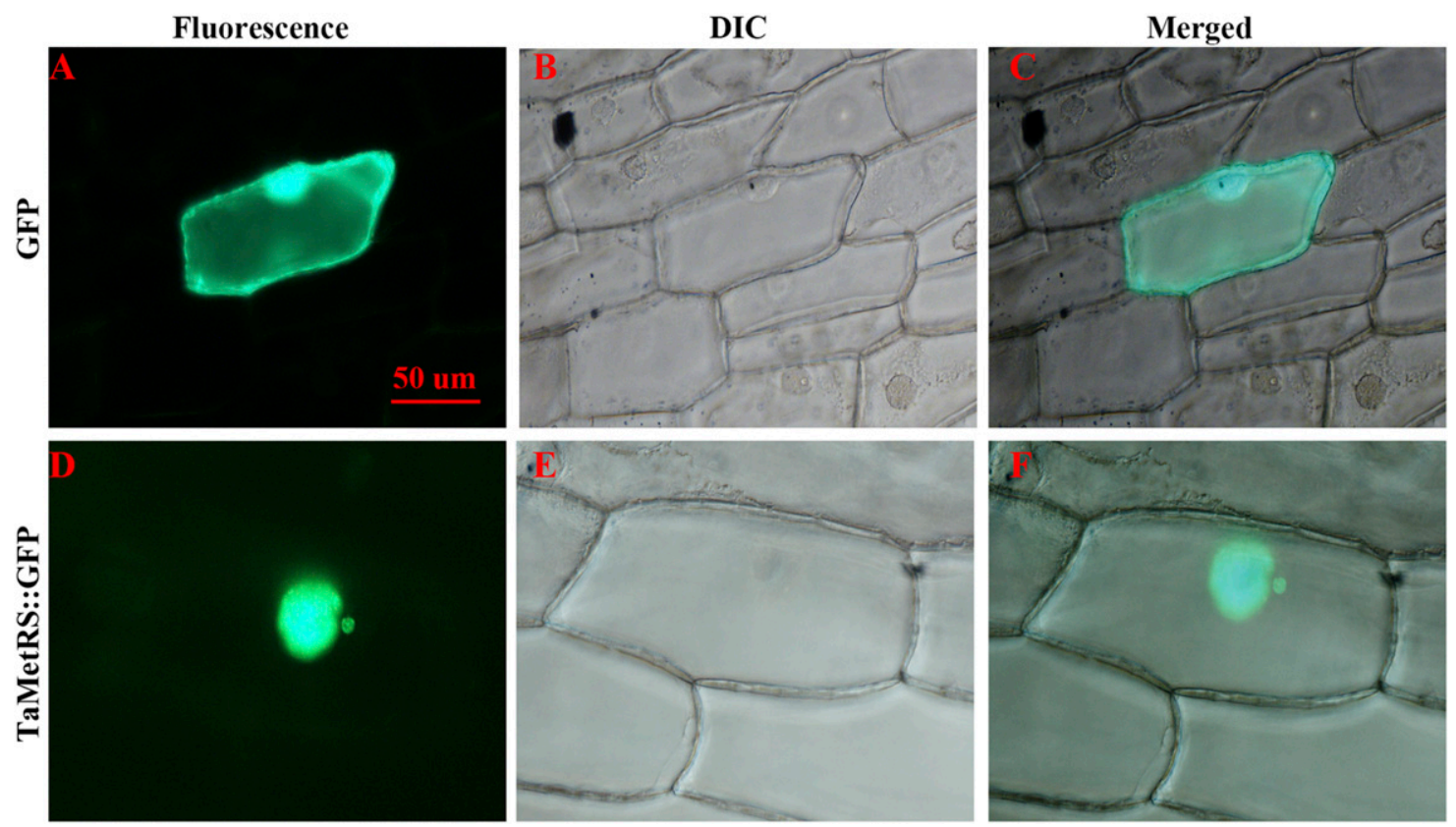

Fig. 4. Subcellular location of TaMetRS in onion epidermal cells by transient expression. A, Onion epidermal cells expressing GFP alone, showing green fluorescent signals in the nucleus, the plasma membrane, and the cytoplasm. B, Differential interference contrast (DIC) image of A. C, Merged image of A and B. D, Onion epidermal cells expressing the TaMetRS::GFP fusion construct showing green fluorescent signals only in the nucleus. E, DIC image of D. F, Merged image of $\mathbf{D}$ and $\mathbf{E}$. Bar, $50 \mu \mathrm{m}$. 
A

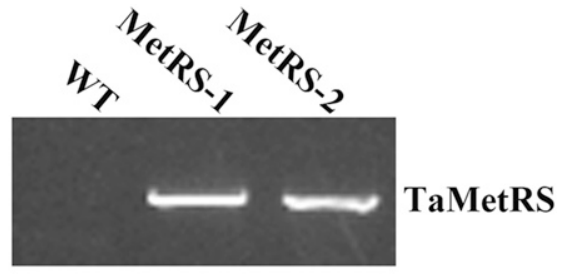

B

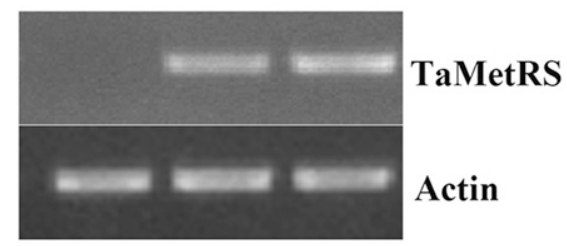

C

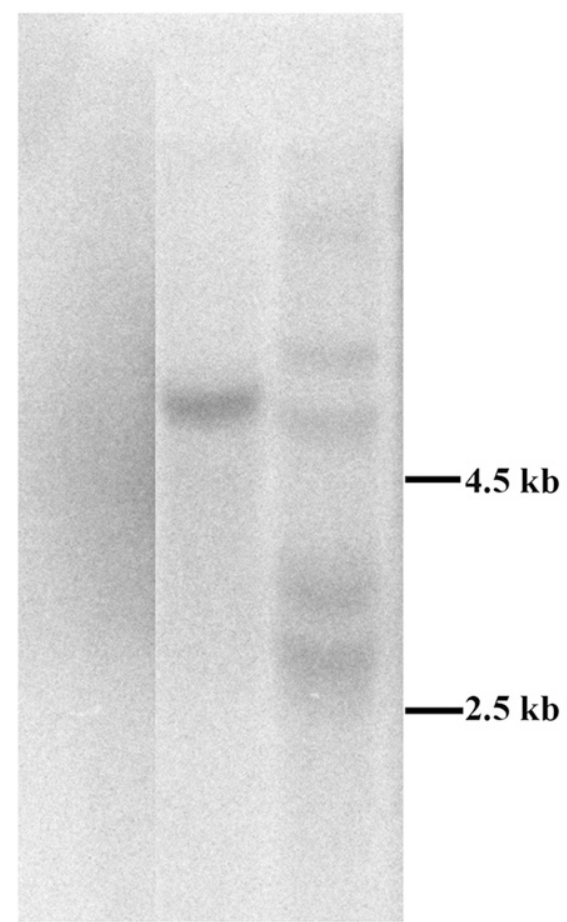

Fig. 5. Transformation and expression of the TaMetRS gene in Arabidopsis thaliana. A, DNA extracted from leaves of T2 transgenic Arabidopsis thaliana lines TaMetRS-1 and TaMetRS-2, and wild-type Arabidopsis thaliana 'Columbia' (WT) was used as the template for PCR with the TaMetRS-F2/TaMetRS-R2 primer pair. B, RNA extracted from the leaves of Arabidopsis plants in A was used for real-time polymerase chain reaction with TaMetRS-F4/TaMetRS-R4 primer pair to detect transcription of the TaMetRS gene; the Actin-F/Actin-R primer pair was used to detect the expression of the $\beta$-actin gene. $\mathbf{C}$, DNA extracted from the leaves of Arabidopsis plants in $\mathbf{A}$ was digested overnight with SacI and electrophoresed on $0.8 \%$ agarose gels, transferred to a Hybond- $\mathrm{N}^{+}$membrane, and probed with a TaMetRS gene probe. The positions of molecular weight markers, in kilobases, are indicated on the right. inhibitory effects of DON in T2 transgenic Arabidopsis line MetRS-1 and nontransgenic control plants after 14 days of DON treatment. An inhibitory effect on root growth was seen for the $5 \mathrm{ppm}$ DON treatment, and this inhibition became more pronounced as the DON concentrations increased. Roots were clearly inhibited at $10 \mathrm{ppm}$, and they were more severely inhibited at $15 \mathrm{ppm}$, giving rise to a substantial difference between the MetRS-1 plants and the controls (Fig. 6). These results indicated that the heterologous expression of a TaMetRS gene in Arabidopsis conferred a strong tolerance to DON, and that clear differences in root growth were observable between the transgenic and nontransgenic control Arabidopsis plants at a DON dosage of $15 \mathrm{ppm}$.

The DON tolerance conferred by the TaMetRS gene was assessed in two independent T2 transgenic Arabidopsis thaliana lines (MetRS-1 and MetRS-2) following treatment with 15 ppm DON. Root lengths and fresh weights of 60 plants per genotype were measured at treatment day 14 . The two transgenic lines had root lengths that were 127 and $186 \%$ longer, respectively, than the roots of the nontransgenic controls (Table 2). The fresh weights of plants of the two transgenic lines were 131 and $86 \%$ greater than the fresh weights of the nontransgenic control plants. Very similar root length and fresh weight results were observed for $\mathrm{T} 3$ generation of transgenic plants (Table 2). These results indicated that the heterologous expression of a TaMetRS gene conferred genetically stable tolerance to DON in Arabidopsis plants.

Fusarium resistance in transgenic Arabidopsis expressing TaMetRS. To further explore the effects of TaMetRS expression in Arabidopsis in the context of fungal disease, wild type control plants and plants from that of the MetRS- 1 and MetRS-2 transgenic Arabidopsis lines were inoculated with the DON-producing 5035 Fusarium strain. The two T2 transgenic lines had FADs of 6.59 and 5.10 at $7 \mathrm{dpi}$, and 8.41 and 5.91 at $10 \mathrm{dpi}$, with significant disease reductions of up to 55 and $57 \%$ at 7 and 10 dpi, respectively, compared with the progression of infection from nontransgenic controls (Table 2). T3 generation transgenic plants showed comparably significant disease resistance to Fusarium pathogens. Typical disease symptoms of transgenic and nontransgenic Arabidopsis plants at 7 and 10 dpi are shown in Figure 7. These results indicated that the TaMetRS gene from wheat provides genetically stable resistance against Fusarium pathogens in Arabidopsis.

\section{DISCUSSION}

Controlling Fusarium pathogens and associated mycotoxins in the production of many staple crops is a challenge due to the limited availability of resistant germplasm. Isolation and functional characterization of novel genes that can confer resistance to mycotoxins and mycotoxin-producing fungi is vitally important for breeding resistant crop varieties that will improve yields and decrease mycotoxin contamination in both food and feed products. The TaMetRS gene described in this study represents a new class of plant genes that were able to confer durable tolerance to the presence of DON and durable

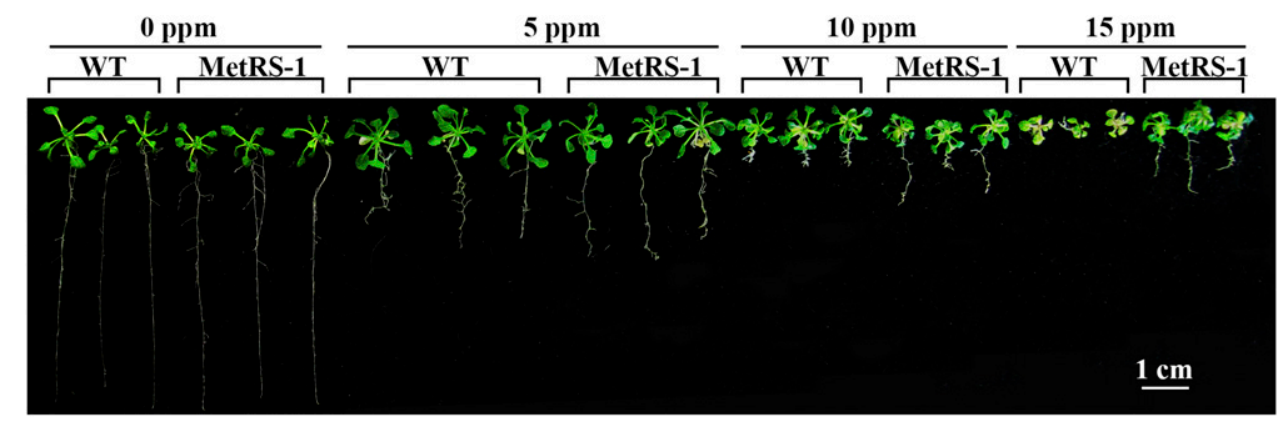

Fig. 6. Deoxynivalenol (DON) tolerance of Arabidopsis thaliana plants. Seedlings of T2 transgenic Arabidopsis thaliana line TaMetRS-1 and wild-type Arabidopsis thaliana 'Columbia' (WT) were grown in media supplemented with 0, 5, 10, or $15 \mathrm{ppm}$ DON. Photographs were taken on the 14th day of treatment. Bar, $1 \mathrm{~cm}$. 
resistance to Fusarium pathogen infection in plants. Therefore, this gene is exciting and has strong potential for further use in both basic and applied research.

The amino acid sequence encoded by the DON-activated TaMetRS gene shared $76 \%$ sequence similarity with a rice MetRS that was previously demonstrated to be located in cytoplasm (Kaminska et al. 2000) and to have function in aminoacylation. TaMetRS contains several characteristic domains that are essential for aminoacylation during protein biosynthesis (Fig. 1). MetRS proteins from many other organisms have nuclear export signals, which imply possible cytoplasmic localization and likely involvement in catalyzing the transfer of Met to cognate tRNA (Galani et al. 2001). However, the TaMetRS investigated in this study contains a putative nuclear localization signal, suggesting translocation into nucleus. We demonstrated using TaMetRS::GFP fusion construct that contains its native NLS together with a transient onion epidermis cell expression system that TaMetRS::GFP was localized to the nucleus. Human MetRS contains putative nuclear localization signals and has been shown to be translocated into the nucleus (Ko et al. 2000), where it plays a role in the biogenesis of ribosomal RNA. Human MetRS has a separate role in catalyzing protein synthesis in the cytoplasm. A recent study reported a new, second function for the human MetRS; it was shown to have tumor suppressor activity while regulating protein translation (Kwon et al. 2011). It seems probable that wheat MetRS may have similar dual roles, and the activated TaMetRS in the nucleus may have a role in biogenesis of ribosomal RNA, as has been reported for human MetRS. DON is known as an inhibitor of protein biosynthesis through interaction with the peptidyltransferase center of eukaryotic ribosomes (Fried and Warner 1981). Protein biosynthesis in wheat is inhibited in the presence of DON. Such a disruption of protein homeostasis may also act as a trigger of gene expression, in which "a feedback rescue regulatory mechanism" may be launched. During this rescue process, the TaMetRS gene is activated and translocated to the nucleus, and the biogenesis of ribosomal RNAs is enhanced. Subsequently, the newly generated ribosomal RNA molecules can be used to form ribosomes, the machinery of protein translation, and thus promote protein biosynthesis to rescue and/or compensate initial inhibition of protein biosynthesis caused by DON. The exact mechanism and regulation involved in the interaction of DON and TaMetRS remains an open question.

The expression patterns of the TaMetRS gene in response to DON appear to support the view that DON is a potent trigger of TaMetRS gene expression. In DON-treated wheat spikes, TaMetRS expression steadily increased from 4 to $24 \mathrm{hpi}$, and then declined after $48 \mathrm{hpi}$ in DON-treated wheat spikes (Fig. 3A), suggesting that stronger expression of TaMetRS gene was activated when more DON molecules entered plant cells. However, such an activation of TaMetRS expression was attenuated when the number of DON molecules in plant cells decreased over time due to metabolization and/or modification by plant cells. This view is further supported by the observed activation of TaMetRS expression only in wheat spikes infected by a DON-producing strain. Further, activation of TaMetRS expression was observed to be dependent on the amount of DON present during fungal colonization (Fig. 3B). DON production typically starts in wheat spikes at around 36 hpi (Jansen et al. 2005; Kang and Buchenauer 1999). In our assays, no induction of TaMetRS expression was seen before $36 \mathrm{~h}$. As the fungal pathogens developed and produced more DON within colonized wheat tissues at $48 \mathrm{hpi}$ and beyond, the expression of TaMetRS increased steadily. In contrast, no changes in TaMetRS expression were observed at any of the time points following infection with the non-DON producing Tri5- strain. Two wheat varieties with varying levels of resistance to Fusarium pathogens (Cheng et al. 2015) showed comparable expression patterns following both the DON treatment and infection with the DON-producing $F$. graminearum strain. These results congruently indicate that the presence of DON causes the activation of TaMetRS expression in wheat, and that this activation occurs in a manner independent of the genetic background of particular wheat varieties.

This exciting genotype-independent, DON-triggered activation of TaMetRS expression in wheat may play a vitally important role

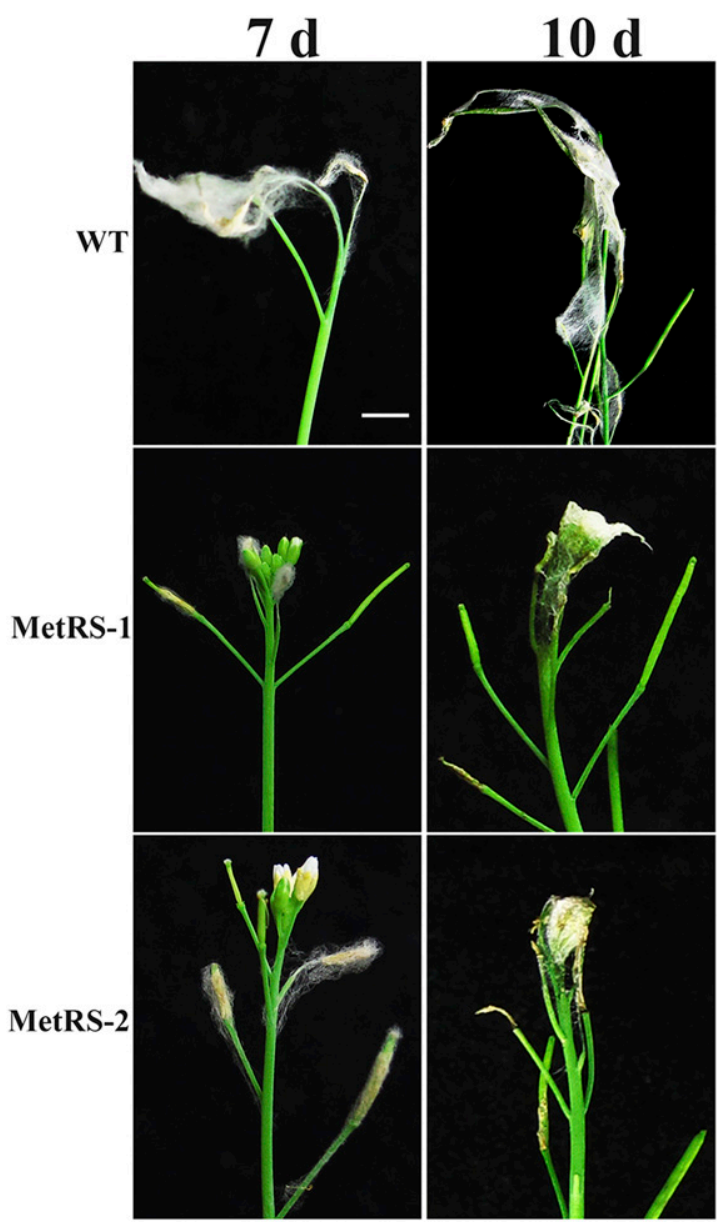

Fig. 7. Phenotype of representative florets from T3 generation transgenic Arabidopsis lines (MetRS-1 and MetRS-2), and wild-type Arabidopsis thaliana 'Columbia' (WT), at 7 and 10 days postinoculation with Fusarium graminearum. Bar, $1 \mathrm{~cm}$.

TABLE 2. Deoxynivalenol tolerance and Fusarium resistance assays of TaMetRS transgenic Arabidopsis plants ${ }^{\mathrm{z}}$

\begin{tabular}{|c|c|c|c|c|c|c|c|c|}
\hline \multirow[b]{3}{*}{ Genotype } & \multirow{2}{*}{\multicolumn{2}{|c|}{ Root length (mm) }} & \multirow{2}{*}{\multicolumn{2}{|c|}{ Fresh weight (mg) }} & \multicolumn{4}{|c|}{ Disease (FAD) } \\
\hline & & & & & \multicolumn{2}{|c|}{$\mathrm{T}_{2}$} & \multicolumn{2}{|c|}{$\mathrm{T}_{3}$} \\
\hline & $\mathrm{T}_{2}$ & $\mathrm{~T}_{3}$ & $\mathrm{~T}_{2}$ & $\mathrm{~T}_{3}$ & 7 days & 10 days & 7 days & 10 days \\
\hline MetRS-1 & $8.50 \pm 1.43 b$ & $10.40 \pm 1.50 b$ & $7.01 \pm 0.40 \mathrm{~b}$ & $5.81 \pm 1.51 \mathrm{~b}$ & $6.59 \pm 2.20 b$ & $8.41 \pm 1.30 \mathrm{~b}$ & $4.14 \pm 1.88 b$ & $5.14 \pm 1.52 b$ \\
\hline MetRS-2 & $10.70 \pm 0.67 b$ & $11.13 \pm 2.56 b$ & $5.65 \pm 1.66 \mathrm{a}$ & $7.87 \pm 1.37 \mathrm{~b}$ & $5.10 \pm 1.97 \mathrm{~b}$ & $5.91 \pm 1.80 \mathrm{~b}$ & $4.18 \pm 1.53 b$ & $4.77 \pm 1.80 \mathrm{~b}$ \\
\hline WT & $3.74 \pm 0.53$ & $4.40 \pm 0.71$ & $3.03 \pm 0.98$ & $4.45 \pm 0.89$ & $11.23 \pm 2.67$ & $13.59 \pm 2.44$ & $7.93 \pm 1.02$ & $9.86 \pm 1.88$ \\
\hline
\end{tabular}

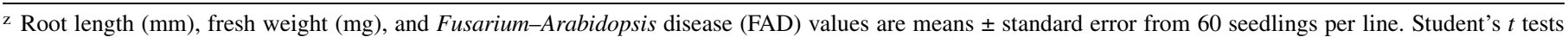
were used to evaluate the data: the letter a at the same time point indicates statistical significance at $P<0.05$, while the letter b indicates statistical significance at $P<0.01$. 
for resistance of wheat varieties to Fusarium pathogens. The interaction between this wheat MetRS gene/gene product and the presence of DON appears to be somewhat similar to a welldocumented interaction system of a MetRS gene from T. brucei and its several inhibitor compounds. In the protozoan $T$. brucei, which causes African trypanosomiasis in human, the TbMetRS protein has been identified as the target of several inhibitors (Ranade et al. 2013). The presence of the inhibitors in the medium induced the expression of the TbMetRS gene. The enhanced expression of TbMetRS gene in T. brucei was shown to be responsible for the observed resistance to inhibitors, and this resistance was verified as durable in subsequent generations (Ranade et al. 2013), indicating that the activated expression is inheritable. Recent crystal structure analyses of TbMetRS revealed that its catalytic domain is formed by Rossmann fold and stem-contact fold domains, where "HIGH" and "KMSKS" motifs are highly conserved (Koh et al. 2012). These conserved structures are all present in the TaMetRS in this study (Fig. 1). Previous studies showed that plants of several different wheat genotypes that were selected and regenerated from tissue culture media containing DON displayed genetically stable, enhanced tolerance to DON and resistance to Fusarium pathogens (Cao and $\mathrm{Yu}$ 1991; Lu et al. 1998). In this light, we assume that the wheat MetRS gene reported here was very likely among the targets activated by DON in the media of those tissue culture selection experiments from the 1990s. If this is indeed the case, it would follow that the DON-activated expression of TaMetRS can be genetically stable and heritable. Obviously, further investigations are required to assess this conjecture.

In summary, we isolated a novel TaMetRS gene from wheat, and found that although the deduced polypeptide of the gene carries characteristic features for aminoacylation in the cytoplasm, the gene product (as a GFP fusion) is localized to the nucleus. The activation of TaMetRS gene expression by DON showed that this mycotoxin acts as a trigger for the expression of a protein biosynthesis gene in eukaryotes; a role appearing to be separate from the known function of DON as an inhibitor of protein biosynthesis. The nuclear localization of TaMetRS and the TaMetRS-mediated tolerance to DON and TaMetRS-mediated resistance to Fusarium pathogens in plants together suggest that TaMetRS may play a signaling or regulatory role that affects the transcription of other genes. The novel gene identified in this study can be used for the improvement of both plant tolerance to DON and plant resistance to Fusarium pathogens. Further, we present an argument based on our empirical data and a consideration of the MetRS literature that suggests TaMetRS may be a new type of eukaryotic MetRS protein, and that it may function in some novel plant response mechanism for defense against fungal pathogens and/or mycotoxins.

\section{ACKNOWLEDGMENTS}

This research was supported by the National Basic Research Program of China (2013CB127801), the National Natural Science Foundation of China (31271717, 31271718, 31272004), and the Ministry of Agriculture of China (2014ZX0800202B-001).

\section{LITERATURE CITED}

Abebe, T., Skadsen, R. W., and Kaeppler, H. F. 2004. Cloning and identification of highly expressed genes in barley lemma and palea. Crop Sci. 44:942-950.

Altschul, S. F., Madden, T. L., Schäffer, A. A., Zhang, J., Zhang, Z., Miller, W., and Lipman, D. J. 1997. Gapped BLAST and PSI-BLAST: A new generation of protein database search programs. Nucleic Acids Res. 25:3389-3402.

Bai, G. H., Desjardins, A. E., and Plattner, R. D. 2002. Deoxynivalenolnonproducing Fusarium graminearum causes initial infection, but does not cause disease spread in wheat spikes. Mycopathologia 153:91-98.

Bai, G. H., and Shaner, G. 2004. Management and resistance in wheat and barley to Fusarium head blight. Annu. Rev. Phytopathol. 42:135-161.

Cao, Q., and Yu, Y. 1991. Immature embryo callus culture and somatic selection to scab resistance in wheat. J. Huazhong Agric. Univ. 10:9-15.

Chen, F. F., Zhang, J. T., Song, X. S., Yang, J., Li, H. P., Tang, H. R., and Liao, Y. C. 2011. Combined metabonomic and quantitative real-time PCR analyses reveal systems metabolic changes of Fusarium graminearum induced by Tri5 gene deletion. J. Proteome Res. 10:2273-2285.

Cheng, W., Li, H. P., Zhang, J. B., Du, H. J., Wei, Q. Y., Huang, T., Yang, P., Kong, X. W., and Liao, Y. C. 2015. Tissue-specific and pathogen-inducible expression of a fusion protein containing a Fusarium-specific antibody and a fungal chitinase protects wheat against Fusarium pathogens and mycotoxins. Plant Biotechnol. J. 13:664-674.

Desjardins, A. E., Proctor, R. H., Bai, G. H., McCormick, S. P., Shaner, G., Buechley, G., and Hohn, T. M. 1996. Reduced virulence of trichothecenenonproducing mutants of Gibberella zeae in wheat field tests. Mol. PlantMicrobe Interact. 9:775-781.

Fellers, J., Guenzi, A., and Taliaferro, C. 1995. Factors affecting the establishment and maintenance of embryogenic callus and suspension cultures of wheat (Triticum aestivum L.). Plant Cell Rep. 15:232-237.

Food and Agriculture Organization. 2004. Worldwide regulations for mycotoxins in food and feed in 2003. FAO food and nutrition paper 81 . Food and Agriculture Organization of the United Nations, Rome, Italy.

Fried, H. M., and Warner, J. R. 1981. Cloning of yeast gene for trichodermin resistance and ribosomal protein L3. Proc. Natl. Acad. Sci. USA 78:238-242.

Galani, K., Großhans, H., Deinert, K., Hurt, E. C., and Simos, G. 2001. The intracellular location of two aminoacyl-tRNA synthetases depends on complex formation with Arc1p. EMBO J. 20:6889-6898.

Goswami, R. S., and Kistler, H. C. 2004. Heading for disaster: Fusarium graminearum on cereal crops. Mol. Plant Pathol. 5:515-525.

Havrylenko, S., Legouis, R., Negrutskii, B., and Mirande, M. 2010. MethionyltRNA synthetase from Caenorhabditis elegans: A specific multidomain organization for convergent functional evolution. Protein Sci. 19:2475-2484.

Ito, M., Sato, I., Ishizaka, M., Yoshida, S., Koitabashi, M., Yoshida, S., and Tsushima, S. 2013. Bacterial cytochrome P450 system catabolizing the Fusarium toxin deoxynivalenol. Appl. Environ. Microbiol. 79:1619-1628.

Jansen, C., Von-Wettstein, D., Schafer, W., Kogel, K. H., Felk, A., and Maier, F. J. 2005. Infection patterns in barley and wheat spikes inoculated with wild-type and trichodiene synthase gene disrupted Fusarium graminearum. Proc. Natl. Acad. Sci. USA 102:16892-16897.

Johnson, D. D., Flaskerud, G. K., Taylor, R. D., and Satyanayana, V. 2003. Quantifying economic impacts of Fusarium head blight in wheat. Pages 461-483 in: Fusarium Head Blight of Wheat and Barley. K. J. Leonard and W. R. Bushnell, eds. The American Phytopathological Society, St. Paul, MN.

Kaminska, M., Deniziak, M., Kerjan, P., Barciszewski, J., and Mirande, M. 2000. A recurrent general RNA binding domain appended to plant methionyl-tRNA synthetase acts as a cis-acting cofactor for aminoacylation. EMBO J. 19:6908-6917.

Kang, Z. S., and Buchenauer, H. 1999. Immunocytochemical localization of Fusarium toxins in infected wheat spikes by Fusarium culmorum. Physiol. Mol. Plant Pathol. 55:275-288.

Kazan, K., Gardiner, D. M., and Manners, J. M. 2012. On the trail of a cereal killer: Recent advances in Fusarium graminearum pathogenomics and host resistance. Mol. Plant Pathol. 13:399-413.

Ko, Y. G., Kang, Y. S., Kim, E. K., Park, S. G., and Kim, S. 2000. Nucleolar localization of human methionyl-tRNA synthetase and its role in ribosomal RNA synthesis. J. Cell Biol. 149:567-574.

Koh, C. Y., Kim, J. E., Shibata, S., Ranade, R. M., Yu, M. Y., Liu, J. Y., Gillespie, J. R., Buckner, F. S., Verlinde, C., Fan, E., and Hol, W. 2012. Distinct states of methionyl-tRNA synthetase indicate inhibitor binding by conformational selection. Structure 20:1681-1691.

Kwon, N. H., Kang, T., Lee, J. Y., Kim, H. H., Kim, H. R., Hong, J., Oh, Y. S., Han, J. M., Ku, M. J., Lee, S. Y., and Kim, S. 2011. Dual role of methionyltRNA synthetase in the regulation of translation and tumor suppressor activity of aminoacyl-tRNA synthetase-interacting multifunctional protein-3. Proc. Natl. Acad. Sci. USA 108:19635-19640.

Li, H. B., Xie, G. Q., Ma, J., Liu, G. R., Wen, S. M., Ban, T., Chakraborty, S., and Liu, C. J. 2010a. Genetic relationships between resistances to Fusarium head blight and crown rot in bread wheat (Triticum aestivum L.). Theor. Appl. Genet. 121:941-950.

Li, H. P., Zhang, J. B., Shi, R. P., Huang, T., Fischer, R., and Liao, Y. C. 2008. Engineering Fusarium head blight resistance in wheat by expression of a fusion protein containing a Fusarium-specific antibody and an antifungal peptide. Mol. Plant-Microbe Interact. 21:1242-1248.

Li, X., Zhang, J. B., Song, B., Li, H. P., Xu, H. Q., Qu, B., Dang, F. J., and Liao, Y. C. 2010b. Resistance to Fusarium head blight and seedling blight in wheat is associated with activation of a cytochrome P450 gene. Phytopathology 100:183-191.

Lu, W. Z., Chen, S. H., Shen, X. R., Zhou, C. F., Zhang, K. J., and Wang, Y. Z. 1998. Study on utilization of cell engineering in breeding wheat for scab resistance. Jiangsu J. Agric. Sci. 14:9-14

Ma, L. L., Shang, Y., Cao, A. Z., Qi, Z. J., Xing, L. P., Chen, P. D., Liu, D. J., and Wang, X. E. 2010. Molecular cloning and characterization of an upregulated UDP-glucosyltransferase gene induced by DON from Triticum aestivum L. cv. Wangshuibai. Mol. Biol. Rep. 37:785-795. 
McMullen, M., Jones, R., and Gallenberg, D. 1997. Scab of wheat and barley: A re-emerging disease of devastating impact. Plant Dis. 81:1340-1348.

Netzer, N., Goodenbour, J. M., David, A., Dittmar, K. A., Jones, R. B., Schneider, J. R., Boone, D., Eves, E. M., Rosner, M. R., Gibbs, J. S., Embry, A., Dolan, B., Das, S., Hickman, H. D., Berglund, P., Bennink, J. R., Yewdell, J. W., and Pan, T. 2009. Innate immune and chemically triggered oxidative stress modifies translational fidelity. Nature 462:522-526.

Okubara, P., Blechl, A., McCormick, S., Alexander, N., Dill-Macky, R., and Hohn, T. 2002. Engineering deoxynivalenol metabolism in wheat through the expression of a fungal trichothecene acetyltransferase gene. Theor. Appl. Genet. 106:74-83.

Peschen, D., Li, H. P., Fischer, R., Kreuzaler, F., and Liao, Y. C. 2004. Fusion proteins comprising a Fusarium-specific antibody linked to antifungal peptides protect plants against a fungal pathogen. Nat. Biotechnol. 22:732-738.

Pestka, J. J. 2010. Deoxynivalenol: Mechanisms of action, human exposure, and toxicological relevance. Arch. Toxicol. 84:663-679.

Placinta, C. M., D’Mello, J. P. F., and Macdonald, A. M. C. 1999. A review of worldwide contamination of cereal grains and animal feed with Fusarium mycotoxins. Anim. Feed Sci. Technol. 78:21-37.

Poppenberger, B., Berthiller, F., Lucyshyn, D., Sieberer, T., Schuhmacher, R., Krska, R., Kuchler, K., Glössl, J., Luschnig, C., and Adam, G. 2003. Detoxification of the Fusarium mycotoxin deoxynivalenol by a UDP-glucosyltransferase from Arabidopsis thaliana. J. Biol. Chem. 278:47905-47914.

Qu, B., Li, H. P., Zhang, J. B., Xu, Y. B., Huang, T., Wu, A. B., Zhao, C. S., Carter, J., Nicholson, P., and Liao, Y. C. 2008. Geographic distribution and genetic diversity of Fusarium graminearum and $F$. asiaticum on wheat spikes throughout China. Plant Pathol. 57:15-24.

Ranade, R. M., Gillespie, J. R., Shibata, S., Verlinde, C., Fan, E., Hol, W. G. J., and Buckner, F. S. 2013. Induced resistance to methionyl-tRNA synthetase inhibitors in Trypanosoma brucei is due to overexpression of the target. Antimicrobiol. Agents Chemother. 57:3021-3028.

Rocha, O., Ansari, K., and Doohan, F. M. 2005. Effects of trichothecene mycotoxins on eukaryotic cells: A review. Food Addit. Contam. 22:369-378.
Scherm, B., Balmas, V., Spanu, F., Pani, G., Delogu, G., Pasquali, M., and Migheli, Q. 2013. Fusarium culmorum: Causal agent of foot and root rot and head blight on wheat. Mol. Plant Pathol. 14:323-341.

Schweiger, W., Boddu, J., Shin, S., Poppenberger, B., Berthiller, F., Lemmens, M., Muehlbauer, G. J., and Adam, G. 2010. Validation of a candidate deoxynivalenol-inactivating UDP-glucosyltransferase from barley by heterologous expression in yeast. Mol. Plant-Microbe Interact. 23:977-986.

Shin, S., Torres-Acosta, J. A., Heinen, S. J., McCormick, S., Lemmens, M., Paris, M. P. K., Berthiller, F., Adam, G., and Muehlbauer, G. J. 2012. Transgenic Arabidopsis thaliana expressing a barley UDP-glucosyltransferase exhibit resistance to the mycotoxin deoxynivalenol. J. Exp. Bot. 63:4731-4740.

Tamura, K., Peterson, D., Peterson, N., Stecher, G., Nei, M., and Kumar, S. 2011. MEGA5: Molecular evolutionary genetics analysis using maximum likelihood, evolutionary distance, and maximum parsimony methods. Mol. Biol. Evol. 28:2731-2739.

Urban, M., Daniels, S., Mott, E., and Hammond-Kosack, K. 2002. Arabidopsis is susceptible to the cereal ear blight fungal pathogens Fusarium graminearum and Fusarium culmorum. Plant J. 32:961-973.

Weigel, D., and Glazebrook, J. 2002. Arabidopsis: A Laboratory Manual. Cold Spring Harbor Laboratory, Cold Spring Harbor, NY.

Xu, X. M., and Nicholson, P. 2009. Community ecology of fungal pathogens causing wheat head blight. Annu. Rev. Phytopathol. 47:83-103.

Yang, L., Van der Lee, T., Yang, X., Yu, D., and Waalwijk, C. 2008. Fusarium populations on Chinese barley show a dramatic gradient in mycotoxin profiles. Phytopathology 98:719-727.

Zhang, J. B., Wang, J. H., Gong, A. D., Chen, F. F., Song, B., Li, X., Li, H. P., Peng, C. H., and Liao, Y. C. 2013. Natural occurrence of Fusarium head blight, mycotoxins and mycotoxin-producing isolates of Fusarium in commercial fields of wheat in Hubei. Plant Pathol. 62:92-102.

Zhang, X., Henriques, R., Lin, S. S., Niu, Q. W., and Chua, N. H. 2006. Agrobacterium-mediated transformation of Arabidopsis thaliana using the floral dip method. Nat. Protoc. 1:641-646. 